\title{
EDITORIAL
}

\section{Evaluation of guidelines for community-acquired pneumonia: a story of confounders, surprises and challenges}

\author{
S. Ewig* and T. Welte ${ }^{\#}$
}

$\mathbf{M}$ aybe guidelines are our fate. With the widespread acknowledgement of the principles of evidence-based medicine as the major basis for medical research, the number of evidence-based guidelines following a predefined methodological approach is steadily increasing. Communityacquired pneumonia (CAP) was one of the first diseases to be covered by guidelines. Since then, in addition to the most authoritative American Thoracic Society (ATS)/Infectious Diseases Society of America (IDSA) [1], European Respiratory Society (ERS) [2] and British Thoracic Society (BTS) [3] guidelines, multiple national guidelines have been released and repeatedly updated. Without doubt, the ongoing discussion among international experts covering diverse specialties has substantially stimulated clinical research. Searching the world literature of the last $50 \mathrm{yrs}$, reviewing and rating every meaningful paper, and putting together the available evidence revealed that there are far more questions than answers regarding even the simplest clinical objectives. Some of these were successfully worked on, with the most impressive result being the derivation and validation of tools for severity assessment as the basis for decisions on treatment settings [4]. But, do guidelines on CAP actually improve routine clinical practice?

Several studies have tried to approach this issue. The populations studied were very different: some included all hospitalised patients [5-12], whereas others included subgroups such as outpatients [13] and intensive care unit (ICU) patients [14]. Most studies were retrospective in design [5-9, $14]$ and one relied on a pre-post intervention design [10]. The selected outcomes usually covered length of hospital stay and mortality [5-11, 14]. Most studies focused on antimicrobial treatment as an intervention variable $[6,8,9,11,14]$, while others evaluated clinical pathways [5]. This heterogeneity of study design means conflicting results are not surprising, although most studies found at least some effect of guideline adherence, most in terms of shorter hospitalisation $[7,10,11]$

*Thorax Center, Clinic for Pneumology and Infectious Diseases, EVK Herne und Augusta Hospital, Bochum. ${ }^{\#}$ Clinic for Pneumology, Hannover Medical University, Hannover, Germany.

STATEMENT OF INTEREST: None declared.

CORRESPONDENCE: S. Ewig, Thoraxzentrum Ruhrgebiet, Kliniken für Pneumologie und Infektiologie, EVK Herne und Augusta-Kranken-Anstalt Bochum, Bergstrasse 26, 44791 Bochum, Germany. Fax: 49 2345172463. E-mail: ewig@augusta-bochum.de but some in terms of mortality [5-9]. One study reported an effect on early mortality [12].

In the current issue of the European Respiratory Journal, two additional contributions to this topic are published $[15,16]$. The study from Italy [15] used a multicentre pre-post intervention design, based on Italian guidelines. It evaluated patients at increased risk of death according to Pneumonia Severity Index class IV and V but excluded ICU patients hospitalised in a nonspecialist internal medicine ward. Treatment failures and mortality were primary end-points. In a total population of 2,847 patients, adherence to guidelines was associated with a significantly lower rate of treatment failures and a nonsignificant trend for a lower mortality [15]. Conversely, a study from Spain [16] used a single-centre observational design, based on the ATS guidelines from 2001. It included all patients with CAP consecutively admitted to a hospital during 1 yr. Primary endpoints included length of stay and mortality. The overall population included 780 patients. In multivariate analysis, adherence to guidelines was significantly associated with a shorter hospital stay. There was also a trend towards lower mortality in the adherence group [16].

Two findings in these studies deserve special attention. In the study by BLASI et al. [15], the overall guideline adherence was exceedingly low and did not increase substantially after guideline implementation (from 33 to $44 \%$ ). These figures are difficult to interpret as they could indicate an extremely high potential for improved outcomes when guidelines were generally accepted. Alternatively, they could point to an intelligent noncompliance, i.e. an evident inappropriateness of guidelines in the specific nonspecialist setting. Thus, the appropriate conclusion may not be that there is a "need for future more aggressive and proactive approaches" as pointed out by BLASI et al. [15], but that the reasons behind nonadherence should be carefully investigated. In the study by DAMBRAVA et al. [16], nonadherence to guidelines in low-risk patients was mainly due to a lack of atypical coverage; however, these pathogens were only rarely identified as causative pathogens. Nonadherence to guidelines in ICU patients was most commonly due to a lack of coverage for Pseudomonas aeruginosa. Ironically, the study by DAMBRAVA et al. [16] provides evidence that this noncompliance was justified as, in contrast to a previous landmark study [17], only very few patients at risk actually had CAP due to P. aeruginosa. This is in line with recent German data from the Community-acquired 
Pneumonia Competence Network (CAPNETZ) study group [18]. Thus, although the pathogen patterns found should not lead to adverse outcomes in the case of nonadherence, this is what was actually found to be associated with longer length of hospital stay and a trend towards higher mortality. Taken together, the impression is that important recommendations in guidelines may be not applicable or even erroneous at the time of their evaluation and that observed effects on outcomes may be due to factors other than antimicrobial treatment.

The studies by BLASI et al. [15] and DAMBRAVA et al. [16] both used adherence to guideline recommendations for antimicrobial treatment as the sole variable to evaluate clinical outcomes. This insinuates that antimicrobial treatment may be the most important variable in determining outcomes, including mortality, which is clearly highly questionable. According to current knowledge, low-risk patients are at a very low risk of death as the impact of antimicrobial treatment on mortality is minimal [4]. The determination of its exact amount would require enormous patient numbers in order to detect potential differences. A mortality benefit in low-risk patients therefore remains highly implausible. Patients at high risk of death from CAP may not benefit from appropriate antimicrobial treatment for three reasons. First, the effects of antimicrobial treatment in severe CAP seem to be highly related to time to adequate antimicrobial treatment [19], which was not recorded in these studies. Secondly, death from severe CAP relates, in part, to genetic host factors that cannot be influenced even by timely administered adequate antimicrobial treatment [20]. Accordingly, one study that specifically addressed ICU patients did not find a benefit from guideline implementation [14]. Finally, one major additional confounder when assessing mortality has not yet been adequately recognised. In our prosperous societies, we see an increasing rate of aged and severely disabled persons in whom CAP (or nursing home-acquired pneumonia) is a frequent pre-terminal event. Some form of treatment limitation is frequently applied in these patients. These limitations may not reach the level of "do not resuscitate" or "allow natural death" orders but may subliminally be applied, e.g. by ordering monotherapy instead of combination antimicrobial treatment or by restricting admission to ICU or intermediate care. Although it is clearly impossible to regularly document such treatment restrictions in all patients prospectively, this should be performed post hoc in order to correct for biases emerging from limitations of care.

It could be argued that the only way to deal with the long list of potential biases is a controlled randomised study. However, such a study would be extremely difficult to perform, mainly due to the virtually impossible task of controlling for either centre effects (in studies randomising hospitals) or intervention effects (in studies randomising patients within a ward). Moreover, taken seriously, this approach would imply that every new release or update of guidelines would need to demonstrate an outcome benefit in a new randomised study.

Evidently, it is time to take a step back. Further studies using comparable study designs within the range of those reported probably will not shed new light on this issue. Instead, we should recognise the difference between the evaluation of treatment recommendations and that of processes of care.
Treatment recommendations can only be evaluated when comparing treatment regimens in well-defined homogeneous groups in a randomised manner, while processes of care can only be compared by standardising treatment interventions. The global evaluation of guidelines finds itself inundated by potential confounders, unable to differentiate what kind of intervention is responsible for an observed effect.

Thus, guideline evaluation should follow a dual approach. One line of research should evaluate different diagnostic recommendations in epidemiological or microbiological studies and therapeutic recommendations in randomised clinical studies. Whatever is found to be superior, the result will always be highly preliminary and tentative recommendation that should not only be subject to permanent re-evaluation but which also must be regarded as guidance never meant to overrule individual clinical decisions. Having said this, assessment of adherence to guideline treatment recommendations would necessarily include a careful analysis of reasons for nonadherence. Only this understanding of adherence can approach the difference between good and bad clinical practice. Another line of research should assess the impact of recommended processes of care on cost and clinical outcomes. For example, only recently could it be shown that standardised order sets, in addition to case managing, can effectively shorten the time to oral antibiotics and reduce the length of hospitalisation [21]. These results are highly relevant not only because of the obvious economic gains but also because such benefits are expected to remain stable after structural implementation of the case-managing concept, even if distinct treatment recommendations may change over time.

In summary, what do the studies by BLASI et al. [15] and DAMBRAVA et al. [16] teach us? First, assessment of clinical endpoints according to guideline adherence is a challenging but difficult task and might not be the best way to evaluate guideline recommendations. Adherence to guidelines was found to be associated with a shorter hospital stay and reduced treatment failures, but it remains uncertain whether this is due to adherence to antimicrobial treatment recommendations. Secondly, the validation of diagnostic and therapeutic recommendations and of recommended processes of care should be performed separately. Thirdly, effects on mortality should carefully differentiate risk groups and no longer be assessed without correction for possible hidden treatment limitations. These objections apply equally to virtually all previously cited studies. The study by BLASI et al. [15] in particular showed an exceedingly high rate of nonadherence in a nonspecialist treatment setting even after guideline implementation. The study by DAMBRAVA et al. [16] resulted in strong arguments against an important guideline recommendation, i.e. regular anti-pseudomonal coverage in patients at risk. Are important sections of the guidelines not applicable or even erroneous after all? Whatever the case, guidelines will only be widely accepted when they are evaluated cautiously, with consideration for the fact that guidelines are no more than a framework subject to continuous revision, forming the background for individual clinical decisions.

\section{REFERENCES}

1 Mandell LA, Wunderink RG, Anzueto A, et al. Infectious Diseases Society of America/American Thoracic Society 
consensus guidelines on the management of communityacquired pneumonia in adults. Clin Infect Dis 2007; 44: Suppl. 2, S27-S72.

2 Woodhead M, Blasi F, Ewig S, et al. Guidelines for the management of adult lower respiratory tract infections. Eur Respir J 2005; 26: 1138-1180.

3 British Thoracic Society Standards of Care Committee. BTS Guidelines for the Management of Community Acquired Pneumonia in Adults. Thorax 2001; 56: Suppl. 4, IV1-IV64.

4 Ewig S, Torres A, Woodhead M. Assessment of pneumonia severity: a European perspective. Eur Respir J 2006; 27: 6-8.

5 Hauck LD, Adler LM, Mulla ZD. Clinical pathway care improves outcomes among patients hospitalized for community-acquired pneumonia. Ann Epidemiol 2004; 14: 669-675.

6 Mortensen EM, Restrepo M, Anzueto A, Pugh J. Effects of guideline-concordant antimicrobial therapy on mortality among patients with community-acquired pneumonia. $A m$ J Med 2004; 117: 726-731.

7 Frei CR, Restrepo MI, Mortensen EM, Burgess DS. Impact of guideline-concordant empiric antibiotic therapy in community-acquired pneumonia. Am J Med 2006; 119: 865-871.

8 Menéndez R, Torres A, Zalacaín R, et al. Guidelines for the treatment of community-acquired pneumonia: predictors of adherence and outcome. Am J Respir Crit Care Med 2005; 172: 757-762.

9 Dean NC, Bateman KA, Donnelly SM, Silver MP, Snow GL, Hale D. Improved clinical outcomes with utilization of a community-acquired pneumonia guideline. Chest 2006; 130: 794-799.

10 Marras TK, Chan CK. Use of guidelines in treating community-acquired pneumonia. Chest 1998; 113: 1689-1694.

11 Menéndez R, Ferrando D, Vallés JM, Vallterra J. Influence of deviation from guidelines on the outcome of community-acquired pneumonia. Chest 2002; 122: 612-617.
12 Mortensen EM, Restrepo MI, Anzueto A, Pugh JA Antibiotic therapy and 48-hour mortality for patients with pneumonia. Am J Med 2006; 119: 859-864.

13 Gleason PP, Kapoor WN, Stone RA, et al. Medical outcomes and antimicrobial costs with the use of the American Thoracic Society guidelines for outpatients with community-acquired pneumonia. JAMA 1997; 278: 32-39.

14 Hirani NA, Macfarlane JT. Impact of management guidelines on the outcome of severe community acquired pneumonia. Thorax 1997; 52: 17-21.

15 Blasi F, Iori I, Bulfoni A, Corrao S, Costantino S, Legnani D. Can CAP guideline adherence improve patient outcome in internal medicine departments? Eur Respir J 2008; 32: 902-910.

16 Dambrava PG, Torres A, Vallès $X$, et al. Adherence to guidelines' empirical antibiotic recommendations and community-acquired pneumonia outcome. Eur Respir J 2008; 32: 892-901.

17 Arancibia F, Bauer TT, Ewig S, et al. Community-acquired pneumonia due to gram-negative bacteria and Pseudomonas aeruginosa: incidence, risk, and prognosis. Arch Intern Med 2002; 162: 1849-1858.

18 Kothe H, Bauer T, Marre R, et al. Outcome of communityacquired pneumonia: influence of age, residence status and antimicrobial treatment. Eur Respir J 2008; 32: 139-146.

19 Kumar A, Roberts D, Wood KE, et al. Duration of hypotension before initiation of effective antimicrobial therapy is the critical determinant of survival in human septic shock. Crit Care Med 2006; 34: 1589-1596.

20 Wunderink RG, Waterer GW. Genetics of communityacquired pneumonia. Semin Respir Crit Care Med 2005; 26: 553-562.

21 Fishbane S, Niederman MS, Daly C, et al. The impact of standardized order sets and intensive clinical case management on outcomes in community-acquired pneumonia. Arch Intern Med 2007; 167: 1664-1669. 